\title{
Onomichi Medical Association (OMA) Method on End of Life Care Management Programs
}

\author{
Hisashi Katayama ${ }^{1,2)}$, Shinya Matsuda ${ }^{3)}$ \\ 1) Onomichi Medical Association \\ 2) Faculty of Medicine, Okayama University \\ 3)Department of Preventive Medicine and Community Health, School of Medicine, University of Occupational \\ and Environmental Health, Japan
}

\begin{abstract}
According to the official estimation, there will be 1.7 million of deaths per year in 2038. It will not possible to prepare the hospital volume enough to accept this volume of deaths, it is indispensible to organize the quality home care system from now. This system must respect the dignity of dying persons and make efforts to assure the precious end-of-life moment with their family. In order to overcome difficulties for continuing end-of-life care at home, clinical support is essential. The Onomichi Medical Association has developed the family doctor system based end-of-life care, so called the OMA method on end of life care management programs. In this article the authors would like to present an overview of this system.
\end{abstract}

Key words: Onomichi Medical Association, End-of-life care, Pain control, Family doctor, Team approach

\section{Introduction}

The place of death for Japanese has been changed dramatically in the last 30 yrs. Proportion of death at hospitals was $52 \%$ in 1980 , but has become substantially increased to $80 \%$ in $2006^{1)}$. During this period, the number of death at hospitals has tremendously increased by 2.3 times. According to Anezaki, if this trend continues, maximum number of death at hospitals is expected to be 1.32 million in 2040, increasing by 460 thousand compared with that in $2006^{2)}$. Anezaki indicated that it would be impossible to have this volume of deaths at hospital mainly by the financial reason. Various factors are considered for the background of increase in death at hospitals. Anezaki indicated that easy access to hospitals and increase of nuclear family are factors contributing to this change.

Place of death is a big problem for individuals. Place of death may correlate with pain and comfort of individuals, and cause individual decision-making

Received: September 16, 2011

Accepted: November 9, 2011

Correspondence: H. Katayama, Katayama Clinic, Kuriharamachi 8513-1, Onomichi, Hiroshima 722-0022, Japan e-mail: miles02katakata@celery.ocn.ne.jp and ethical problems. Aside from these individual problems, rapid aging associates place of death with medical care system problems.

According to the official estimation, there will be 1.7 million of deaths per year in $2038^{3}$ ). As it will not be possible to prepare the hospital volume enough to accept this volume of deaths, it is indispensible to organize the quality home care system from now. This system must respect the dignity of dying persons and make efforts to assure the precious end-of-life moment with their family. One of the most important factors to realize this condition is pain control. Thus clinical support is essential. In order to realize this system, the Onomichi Medical Association (OMA) decided to integrate the end-of-life care into the family doctor based primary care system, so called the OMA method on end of life care management programs. In this article the authors would like to present an overview of this system.

\section{OMA method on end of life care management programs (OMA-EOLC)}

Figure 1 shows the principle of OMA-EOLC. In 2009 when the OMA decided to integrate the palliative 


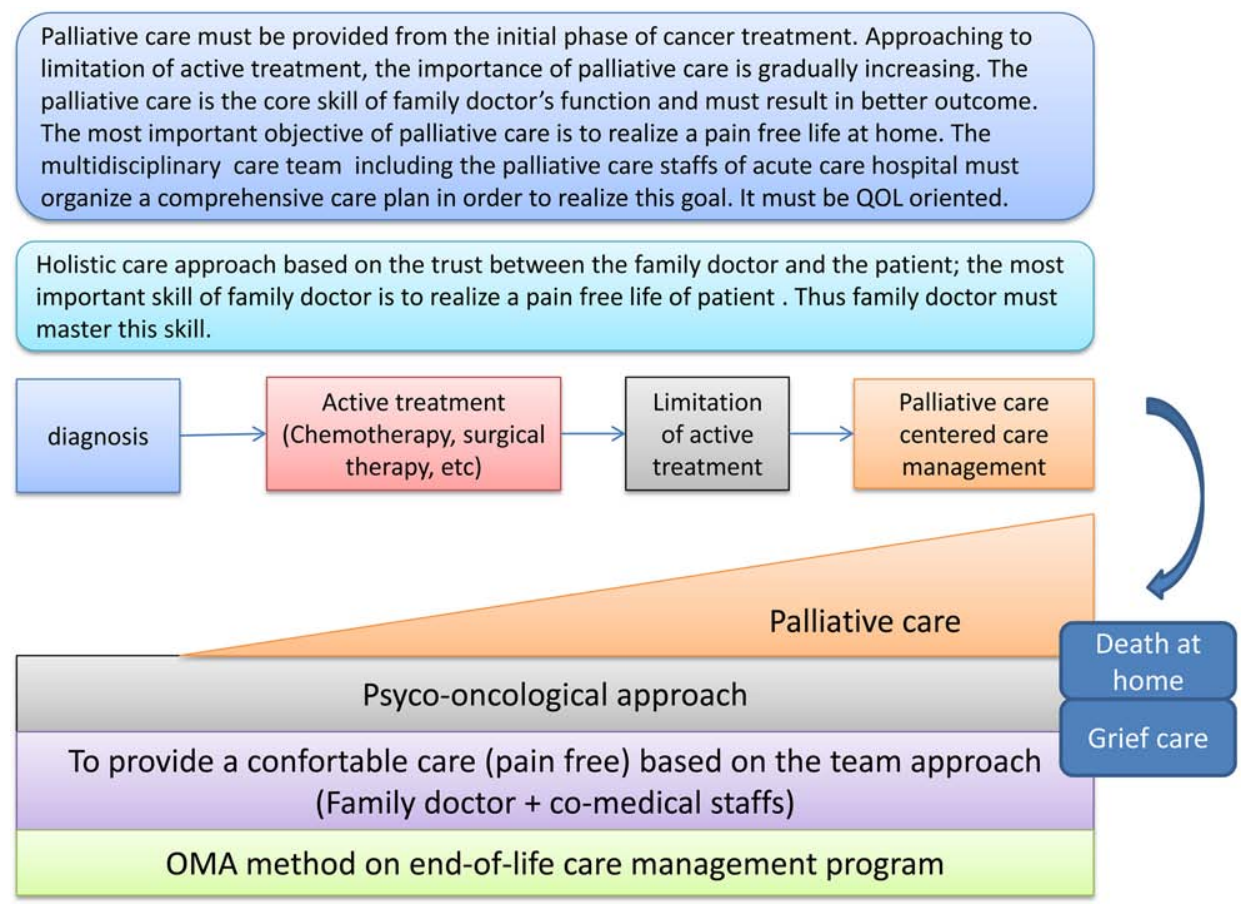

Figure 1 Basic strategy of OMA method on end-of-life care.

care into our family doctor based primary care, we have established the principle of our end-of-life care. The charter indicates; "Palliative care must be provided from the initial phase of cancer treatment. Approaching to limitation of active treatment, the importance of palliative care is gradually increasing. The palliative care is the core skill of family doctor's function and must result in better outcome. The most important objective of palliative care is to realize a pain free life at home. The multidisciplinary care team including the palliative care staffs of acute care hospital must organize a comprehensive care plan in order to realize this goal. It must be QOL oriented". The basis of OMA-EOLC system is the trust between family doctor and patient (including his/her family members).

Figure 2 shows the overview of OMA-EOLC. The pre-discharge care conference organized by the hospital palliative care team and family doctor's team is the basis of this network" (Figure 3). We respect the patient's wish for end-of-life care. In order to do so, in principle a patient and family members are requested to attend the pre-discharge conference held at the acute care hospital. If the patient wishes to receive active treatments, we will arrange so even though the patient in the advanced cancer stage. With the consensus of the patient and his/her family, the treatment is chosen. Our team discusses the expected scenario of prognosis and prepares the care schedule. Just after the discharge, usually semi-acute care will be organized under the collaboration between hospital staffs and family doctor's team, i.e., adjuvant chemotherapy and active pain control using pharmaceuticals. A family doctor's team periodically visits patients home (Figure 4). At the end-of-life stage, family doctor's team will take a pivotal role of palliative care, mainly pain control and onco-psychological support. Sometimes, we will arrange an "end-of-life ceremony" for the patient and family in order to make them to accept it with deep sense of satisfaction (Figure 5). After the patient death, family doctor's team offers a grief care also (Figures 6,7).

The OMA conducted a research concerning the actual situation on palliative care activities among the OMA members. About $74 \%$ of members had experiences of palliative care for their clients. It is important to know that $18 \%$ of members conducted their palliative care with support from other members. This flexibility is one of the characteristics of OMA system.

\section{* Conclusion}

There have been a number of discussions and researches about places of death. Unfortunately most of them have argued it from an economic point of view. 


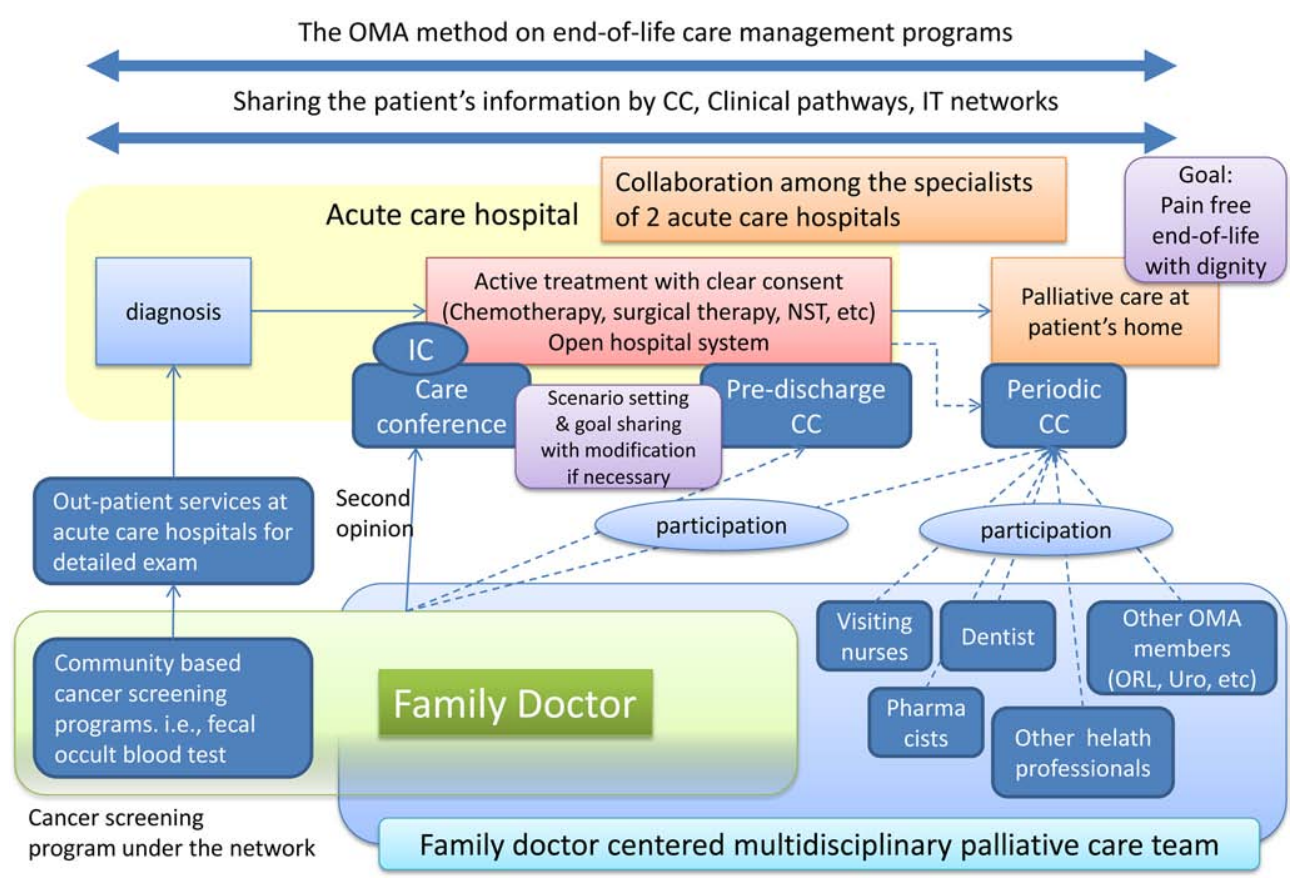

Figure 2 An overview of OMA method on end-of-life care.

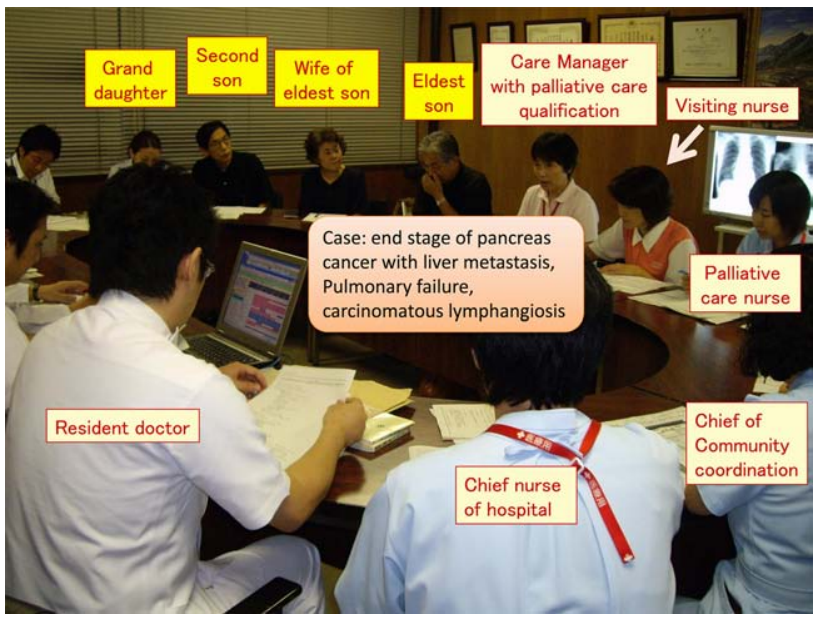

Figure 3 Meeting of the hospital palliative care team and family doctor's team (Care conference at the acute care hospital).

Main question is; "what is the factor of reducing the number of death at hospitals?" Sadamura has clarified that an investment of home care service resource is associated with the low proportion of death at hospitals ${ }^{4}$. Further, Miyashita has clarified that a positive correlation exists between the death rate of natural causes and the proportion of home death ${ }^{5)}$. Yang demonstrated that older patients and males were more likely to die at home ${ }^{6}$. . These literatures have showed

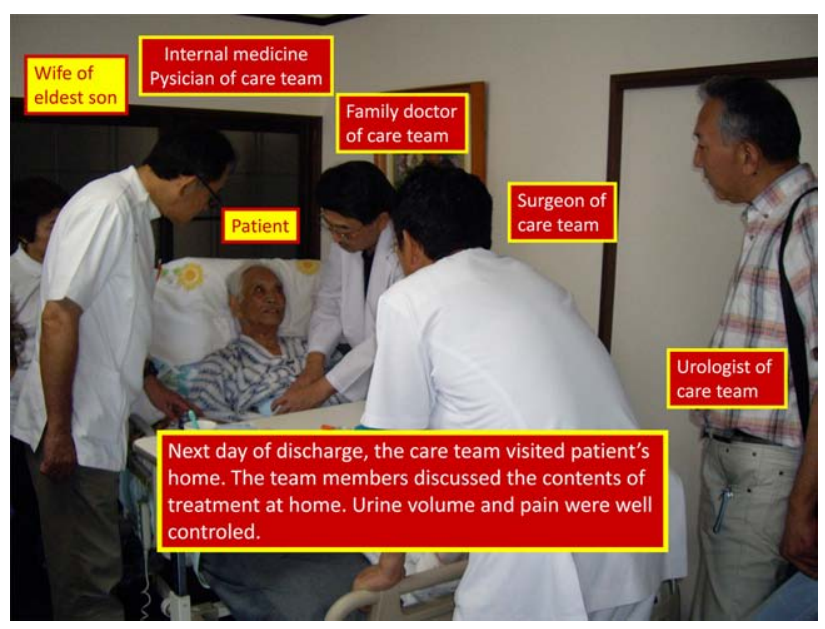

Figure 4 Visit of family doctor's team at patient's home.

that improvement of home care service for elderly is necessary to reduce the proportion of death at hospitals.

In the case of OMA-EODC, we do not organize the home based palliative care system in order to reduce the number of hospital deaths. It will be a byproduct of our activities. Our main purpose is to respond to the wish of our clients. They wish to die at their residence in order to have a finale of their precious lives with their loving family and with dignity. The most important mission of our activities is to medically and psychologically support them to realize 


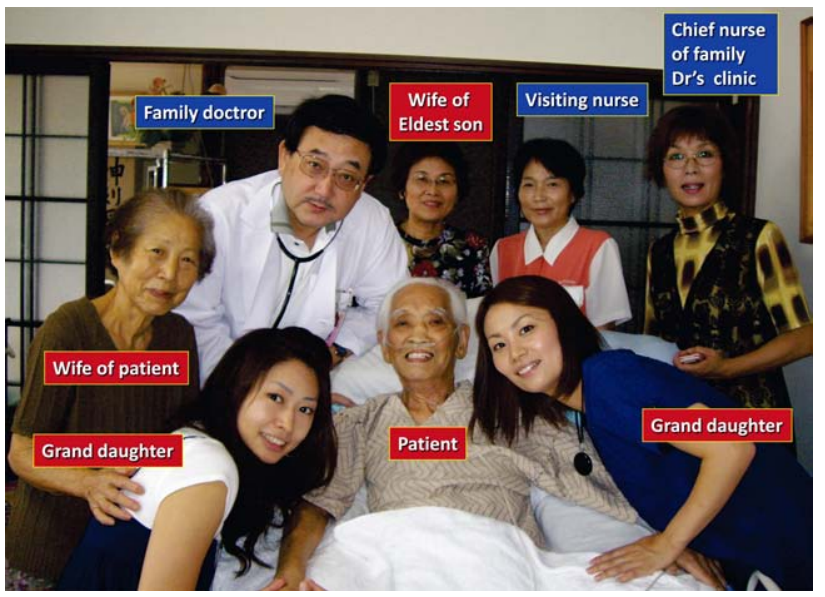

Figure 5 An "end-of-life ceremony" for the patient and family organized by Family doctor's team (cerebration of $88 \mathrm{yr}$ old birthday at patient's home).

Pain was well controlled. Respiratory condition was maintained by $12 \mathrm{~L} / \mathrm{min} \mathrm{O}_{2}$.

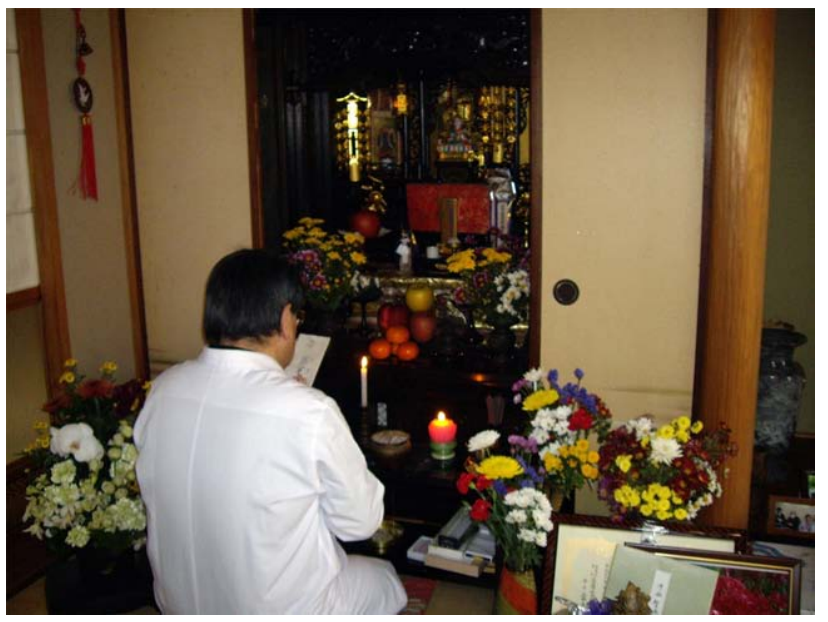

Figure 6 An example of Grief care by the Family doctor.

Two days after the cerebration of 88 yr old birthday, the patient died peacefully at home. Many family members could attend the end of life of the patient.

their wishes. This is the reason why the OMA members can receive a trust from the community members.

Under the highly aged society, the importance of this kind of community care activities will increase. The distinction among hospital, clinic and home based care will be meaningless. The ability to flexibly organize the palliative care using various community resources is important. The family doctor must play a pivotal role for coordination.

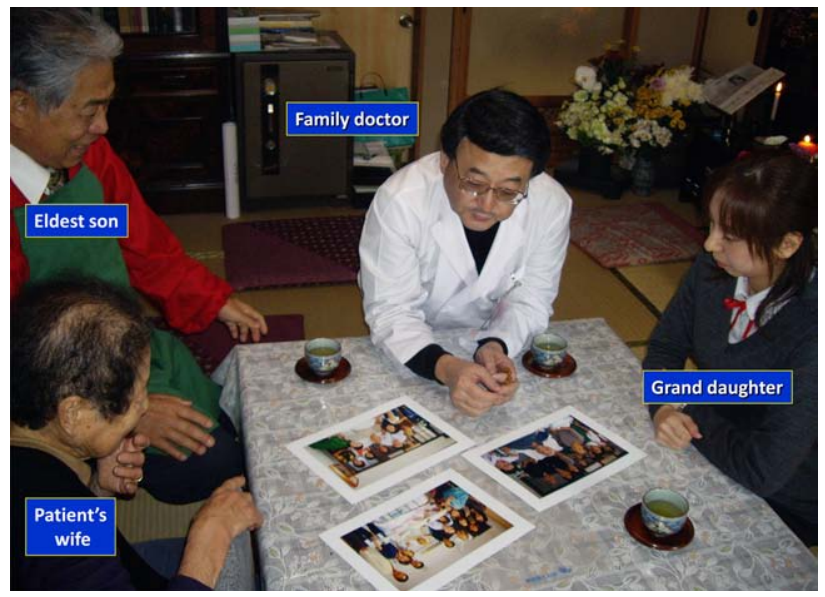

Figure 7 An example of Grief care by the Family doctor. The family members of died patient and family doctor are talking about the patient with patient's photos. The experience of caring during the dying period with lots of affectation could give them a sense of satisfaction.

\section{References}

1) Ministry of Health, Labour and Welfare: Vital statistics. http://www.mhlw.go.jp/toukei/saikin/hw/ jinkou/suii01/deth5.html. (access 23 August 2011).

2) Anezaki T: Transition of the place of death and total number of death in Japan. APJDM, 2, 97-101 (2008).

3) National Institute of Population and Social Security Research: Estimation of future population of Japan. http://www.ipss.go.jp/pp-newest/j/newest02/1/ suikei_g.html. (access 23 August 2011).

4) Sadamura M, Babazono A: Kaigohokenseido ni yoru kaigosigen no shihyou to shiboubasho tono kanren(Correlation between Index of elderly care resource on elderly care insurance system and place of death). Journal of Health and Welfare Statistics (Koseinoshihyo) 52, 8-14 (2005) (in Japanese).

5) Miyashita M, Shirai Y: 2004nen no todouhukenbetsu zaitakusibouwariai to iryou-shakaitekisihyo no kanren (Proportion of place of death in all prefectures and social/medical indicator at 2004). Journal of Health and Welfare Statistics (Koseinoshihyo) 54, 44-49 (2007) (in Japanese).

6) Yang L, Sakamoto N, Marui E: A study of home deaths in Japan from 1951 to 2002. BMC Palliateve Care 5, 1-9 (2006). 